\title{
ANÁLISIS DE LA INFLUENCIA DE LA CONSTRUCCIÓN DE MAPAS CONCEPTUALES SOBRE LA ESTRUCTURA COGNITIVA EN ESTUDIANTES DE FÍSICA ${ }^{(*)}$
}

\author{
LÓPEZ RUPÉREZ, F. \\ Instituto Español de París. 38, Bd. Victor Hugo. 92200 Neuilly Sur Seine.
}

(*) Este trabajo forma parte del proyecto "Organización del Conocimiento y Resolución de Problemas en Física» financiado por el CIDE con cargo al programa de Ayudas a la Investigación Educativa 1987. Ministerio de Educación y Ciencia.

\section{SUMMARY}

In this work we investigated the effect of systematic hierarchical concept mapping by students in a COU (High School) Physics class on their cognitive structure. The results show no statistically significant influence of the treatment, but rather of personal study habits on the number of response words. The results are discussed and constraints to the research procedure used are discussed.

\section{INTRODUCCIÓN}

Los mapas conceptuales constituyen una forma de representación bidimensional de un sistema conceptual y de sus relaciones internas. Debido a su propia naturaleza, la idea de mapa conceptual ha sido utilizada como recurso para representar tanto la estructura cognitiva de los estudiantes como la estructura lógica de la disciplina (Lopez Rupérez 1990). La orientación dada por Novak -pionero en el uso de dicho término (Novak 1976)-a los referidos mapas se inserta en la teoría de Ausube: del aprendizaje significativo a modo de desarrollo tecnologico (Novak et al. 1984, Novak 1985, 1988). A pesar de su reconocida popularidad pocas veces se ha puesto a prueba su eficacia a la hora de mejorar significativamente eI aprendizaje científico; incluso las veces que se ha hecho ha sido considerando los mapas conceptuales como un elemento más dentro de un sistema instructivo completo de corte ausubeliano (Novak et al. 1983, Bascones tet al. 1985). El propio Novak, en su trabajo junto con Bascones (Bascones et al. 1985), reconoce que «debería realizarse investigación adicional en orden a contestar a la siguiente cuestión: ¿Cuál de los elementos del sistema instructivo tiene mayor poder predictivo respecto del éxito en la resolución de problemas?» (p. 261).
La presente investigación forma parte de un proyecto más amplio que se planteo, entre sus objetivos, cl evaluar qué añade por sí misma la construcción de mapas conceptuales de tipo jerárquico, en tanto que herramienta para promover la organización del conocimiento declarativo, en la efectividad en la resolución de problemas de Física característicos de un nivel de instrucción preuniversitario (López Rupérez et al. 1989). El conocimiento declarativo -a veces denominado conceptual, proposicional, o verbal- constituye una componente indiscutible del conocimiento científico y es condición necesaria para la resolución de problemas con carga semántica. Por tal motivo, en el contexto de nuestra investigación, nos pareció oportuno efectuar un análisis empirico del efecto ejercido sobre la estructura cognitiva del alumno por la construcción del mapa conceptual. A diferencia de estudios anteriores en los que se ha optado por un diseño, ya sea de tipo experimental (Moreira et al. 1985 , Novak et al. 1983) ya sea de tipo correlacional (Novak et al. 1983) que se apoya en alguna forma de calificación de los mapas -cuya validez es cuando menos dudosa (Stuart 1985)-, en el presente estudio, hemos recurrido a un diseño experimental en el que la variable 
dependiente fuese medida con instrumentos ajenos al propio mapa, como lo son los tests de asociación de palabras, una herramienta que aun cuando no está exenta de detractores (Strike et al. 1976, Stewart 1979) ha sido empleada ampliamente en el contexto de la educación científica (Shavelson 1972, 1973, 1974, Preece 1976, 1978, Thro 1978, Gunstone 1980, Moreira et al, 1981, Moynihan 1981, 1982, Kempa 1983, 1986, Johnstone et al. 1985, Gorodetsky et al. 1986, Gussarsky et al. 1988)(1). Ello nos ha permitido aislar, dentro de lo posible, el efecto del tratamiento y mejorar, respecto de otras investigaciones, la validez interna del experimento.

\section{METODOLOGÍA}

\section{Muestra}

El procedimiento de selección de la muestra sobre la que se cfectuó el presente estudio empírico fue de tipo incidental, si bien se tuvieron en consideración algunos elementos de control muestral. Así, tras una exposición de los elementos esenciales de la investigación, de sus fines y de sus procedimientos dirigida a los alumnos de COU de Ciencias del Instituto Español de París (curso escolar 1987-88) se constituyó la muestra efectiva, formándose ésta únicamente por alumnos comprometidos, de un modo voluntario, con la experiencia. Los 18 estudiantes así reclutados, se agruparon en tres niveles de clasificación de acuerdo con los resultados del rendimiento en Física obtenidos a lo largo del primer trimestre. Cada nivel de calificación contenía seis alumnos de rendimiento análogo entre los cuales se efectuó una asignación al azar al grupo experimental o al grupo de control. De este modo, y dada la limitada extensión de la muestra $(N=18)$, pretendimos reducir el posible sesgo introducible mediante una asignación por simple azar.

\section{Hipótesis}

Formuladas en términos positivos, las hipótesis conductoras del presente estudio fueron las siguientes:

1. Iil trabajo personal del alumno, como etapa decisiva previa a la realización đe un examen, debe traducirse en un enriquecimiento de su estructura cognitiva (asociativa) medida mediante tests de asociación de palabras.

2. I.a confección personalizada, por parte del alumno, de mapas conceptuales, como instrumento de organización del conocimiento conceptual, debe mejorar la estructura cognitiva (asociativa) medida mediante este tipo de pruebas.

\section{Instrumentos y variables}

Los instrumentos de medida empleados en el presente cstudio consistieron en una serie de tres tests de asociación de palabras, correspondientes, cada uno de ellos, a los temas trabajo y energía mecánica, dinámica del sólido rígido y campo eléctrico, respectivamente. El Anexo 1 muestra las tres series de epalabras estímulon relativas a dichos temas. Con ocasión de la primera de estas pruebas, los alumnos recibieron la hoja de instrucciones que se muestra en el Anexo 2. Siguiendo a Shavelson (1972) se concedieron a los alumnos intervalos sucesivos de un minuto para producir «palabras respuesta» asociadas con cada una de las palabras estímulo. En el presente estudio se ha elegido como variable indicadora de la asociación conceptual de los alumnos, el número de palabras respuestas que están relacionadas con la palabra estímulo mediante alguna ecuación o cadena de ecuaciones. La selección de esta variable y no de otras -como el número total de palabras respuestas emitidasviene recomendada por los resultados obtenidos en estudios previos en los cuales la conexión con otras variables relevantes al aprendizaje de la Física se ha revelado más fuertemente significativa(Shavelson 1974); pero además, la orientación dada en la presente investigación a los mapas conceptuales como instrumento de enseñanza/ aprendizaje se acopla perfectamente a una explicitación - establecida, desde Iucgo, a partir de criterios pedagógicos- de la estructura conceptual de la Física, o de una porción limitada de ella, en su dimensión operacional. Parecía, entonces, razonable adecuar al máximo posible la definición del efecto medible a la naturaleza del tratamiento. Por otra parte, se trata de un criterio de selección que puede ser aplicado, por lo general sin ambigüedad, por un profesor de Física.

Dado que se aplicaron tres pruebas diferentes y cada una de ellas en dos ocasiones sucesivas, representamos la variable efecto en la forma $\mathrm{Nij}$, donde el primer subíndice designa el índice de la prueba $(i=1,2,3)$ y el segundo designa el instante, anterior $(j=1)$ o posterior $(j=2)$ al tratamiento, en el que la prueba fue aplicada.

El recurso a otras variables más sofisticadas como los coeficientes de relacionabilidad o las distancias semánticas (Shavelson 1972, Thro 1978), tan frecuentes en el análisis de resultados de Ios tests de asociación de palabras, fue ignorado provisionalmente en nuestra investigación en tanto las hipótesis planteadas, y particularmente la segunda, no fueran contrastadas. Sólo si se demostraba que el tratamiento modificaba de una forma significativa Ia estructura conceptual (asociativa) de los estudiantes tendría sentido proceder, entonces, a una exploración más fina de dicha estructura asociativa.

\section{Tratamiento}

Comoen otras investigaciones (López. Rupérez 1989), el propósito central del tratamiento en el presente estudio fue promover, de un modo sistemático en tos alumnos del grupo experimentai, una organización jerárquica y, en cierta medida, funcional del conocimiento, recomendación postulada desde posiciones teóricas diferentes (Gagné 1971, Novak et al. 1984, Eylon et al. 1984).

Como herramienta para la organizacion del conocimiento recurrimos a una modalidad de mapas conceptuales que pone el acento en la estructura lógica de la disciplina (López. Rupérez 1987). Así, adoptamos un principio de jerarquización conceptual que sitúa en la parte superior 
del mapa Ios conceptos primitivos, de acuerdo con el enfoque didáctico elegido. Dicho principio, que en un sentido estricto difiere del de inclusividad conceptual, característico de los mapas tipo Novak, puede dar lugar, a partir del segundo y, en ocasiones, del primer nivel jerárquico, a representaciones análogas a las de aquéllos si se toman como primitivos conceptos muy generales en el sentido de muy inclusivos. La ventaja de este tipo de mapas estriba, esencialmente, en facilitar el proceso de construcción conceptual por parte del alumno, desde la perspectiva de la lógica de la disciplina y, en particular, en aquellas situaciones, tan frecuentes en un curso de Física, en la que se han de introducir los llamados conceptos por definición. La funcionalidad del mapa se intentó potenciar incluyendo en los nodos no sólo los conceptos sino también su expresión operacional. Con el fin de evitar un uso abusivo del operacionalismo se pretendió inducir al alumno a la reflexión sobre la naturaleza epistemológica de las relaciones entre los diferentes conceptos incorporados al mapa. Estos ingredientes teóricos se tradujeron en las normas de elaboración del mapa que se resumen en el Anexo 3.

El tratamiento se extendió a lo largo del semestre sobre el que se desarrolló la investigación y comprendió las siguientes acciones:

a) Una exposición a los alumnos, con fines proactivos, sobre la importancia de la organización del conocimiento como etapa preliminar en la resolución de problemas.

b) Una presentación de los mapas conceptuales como instrumento para la organización jerárquica, y en cierta medida, funcional del conocimiento acompañada de una descripción detallada de las normas de elaboración $y$, posteriormente, de una aplicación colectiva, orientada por el profesor, de dichas normas a la construcción de un mapa concreto, que en el presente caso, fue el correspondiente al tema Dinámica de la partícula (López Rupérez. 1987).

c) Al finalizar cada tema y justo antes de la prueba correspondiente, elaboración obligatoria por parte de cada alumno del mapa conceptual relativo al tema en cuestión y presentación posterior al profesor para su valoración.

d) Estímulo continuado por parte del profesor a los alumnos resaltando la importancia y la utilidad de este tipo de herramientas de aprendizaje.

\section{Diseño experimental y técnicas de análisis}

El diseño experimental, orientado por las hipótesis de partida, se atuvo a un modelo simple pretest-postest con grupo de control (Campbell et al. 1973) del tipo:

\section{$\mathrm{RO} 1 \times \mathrm{O} 2$}

\section{$\mathrm{RO}^{\prime} 1 \quad \mathrm{O}^{\prime} 2$}

con la salvedad de que la aleatorización (R) de los grupos experimental y de control ha sido efectuada dentro de estratos de rendimiento inicial equivalente.
Así, justo antes de dar por terminado cada tema de Física de los tres considerados, se les aplicó el mismo test de asociación de palabras a los estudiantes del grupo experimental y a los del grupo de control, dando lugar a las observaciones $O I$ y $O^{\prime} I$ respectivamente, medidas a través de las variables $\mathrm{Nil}(\mathrm{j}=1,2 \circ 3)$. Las observaciones $\mathrm{O} 2$ y O'2 se produjeron posteriormente como preámbulo a los exámenes correspondientes a los respectivos temas y dentro de la propia sesión de examen. Para el grupo de control, la variación que puede derivarse de la comparación entre la observación O'1 y O'2 será atribuible a la actividad desarrollada por el alumno y, eventualmente, por el profesor, en orden a preparar el referido examen. En el caso del grupo experimental para interpretar las diferencias entre $\mathrm{O} 1$ y $\mathrm{O} 2$ habrá que tomar, además, en consideración el efecto del tratamiento (X), esto es, la elaboración personalizada, por parte del alumno, del mapa conceptual sobre el contenido del correspondiente capítulo a examen. Las medidas relativas a este segundo instante corresponden a las variables $\mathrm{Ni} 2(\mathrm{i}=1,2 \circ 3)$

Como técnicas de análisis se emplearon, en cada una de las situaciones de medida, sendas versiones de la $t$ de Student (Serramona 1980) para la comparación de medias referidas a muestras pequeñas $(n<30)$ y relativas, bien a muestras independientes, si se trataba de comparar el grupo experimental con el de control, o bien a muestras apareadas, si se pretendía comparar, para cada uno de estos dos grupos separadamente considerados, el antes con el después.

Aun cuando un análisis de covarianza, en el que se considerara como covariable las puntuaciones de los pretest, aumentaría la sensibilidad estadística de la prueba, nos ha parecido conveniente, dado el carácter reducido de la muestra, optar por este test más grosero o «conservador» antes que arriesgarnos a incurrir en los «falsos positivos» (Campbell et al. 1973 p. 52). No obstante cuando se efectúa dicho tipo de análisis estadístico no se obtienen, en este caso, conclusiones diferentes de las que presentaremos más adelante ${ }^{(2)}$.

\section{RESULTADOS}

Los resultados obtenidos en las diferentes aplicaciones de los tests de asociación de palabras, junto con sus fechas respectivas, se presentan en la tabla I en donde figuran, además, las iniciales de los alumnos que participaron en el experimento así como las calificaciones en Física relativas al primer trimestre. Lo esencial de Ia información cuantitativa recogida en la referida tabla se muestra en la figura 1 en donde se representan el número de palabras respuesta -seleccionadas de acuerdo con la condición anteriormente definida-y su variación con el tiempo para cada una de las pruebas, estableciéndose una distinción explícita entre el grupo experimental y el de control. La tabla 2 recoge los resultados derivados de los análisis estadísticos efectuados sobre los datos de Ia tabla I; en ella se presentan, en cada caso, los valores de la $t$ de Student resultante, del número de grados de libertad (df) y del nivel de significación estadística (p). 
TABLA 1

Resultados correspondientes a las distintas aplicaciones de los tests de asociación de palabras. Los números representados por $\mathrm{Nij}$ indican número total de «palabras respuesta" Jelacionadas con la "palabra estínulos mediante una ecuación o serie de ecuaciones en cada una de las aplicaciones de los diferentes tests. Si representa la variacion observada, en calda caso, entre el antes y el después del tratamiento. La calificación recoge la valoración del rendimiento de los diferentes alumnos durante el primer trimestre académico previo a la realización del experimento.

GR. SLJETO CALIF N11 N12 $\delta 1 \quad$ N21 N22 k2 $\quad$ N31 N32 83

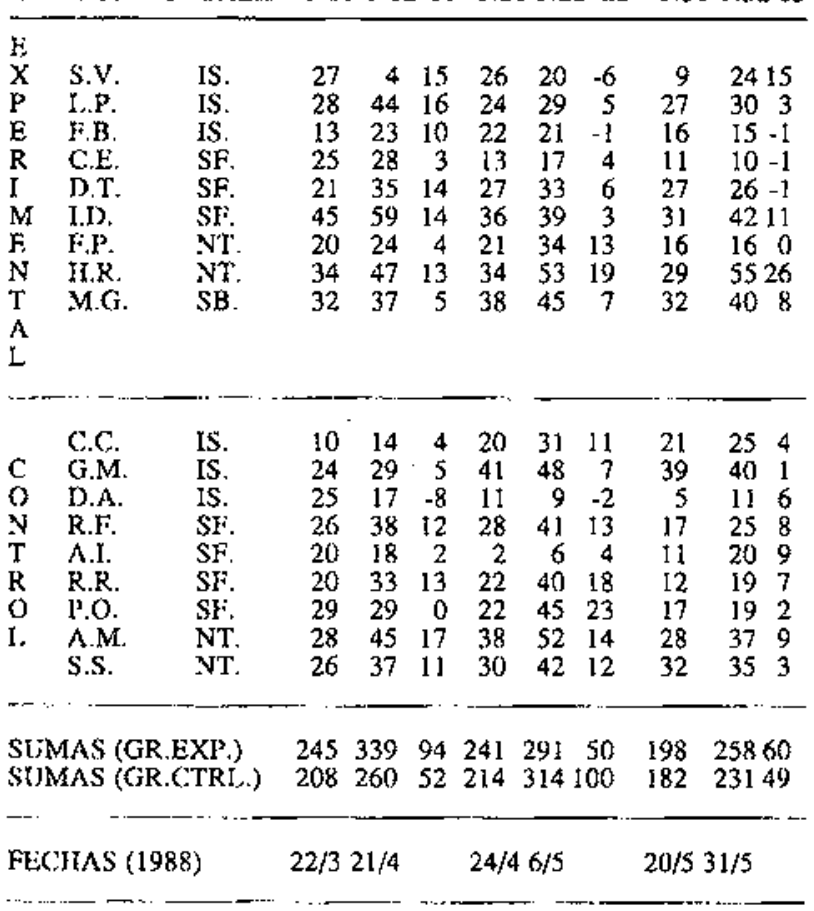

$\mathrm{IS}=$ Instificiente

$\mathrm{SF}=$ Suficiente

NT $=$ Notable

$\mathrm{SB}=$ Sobresaliente
TABLA 2

Resultados del análisis estadístico de los datos recogídos en la tabla 1. Las dos primeras líneas corresponden a la aplicación de la t de Student para muestras dependientes o apareadas, cn tanto que las dos últimas corresponden a la aplicación de la t de Student para muestras independientes.

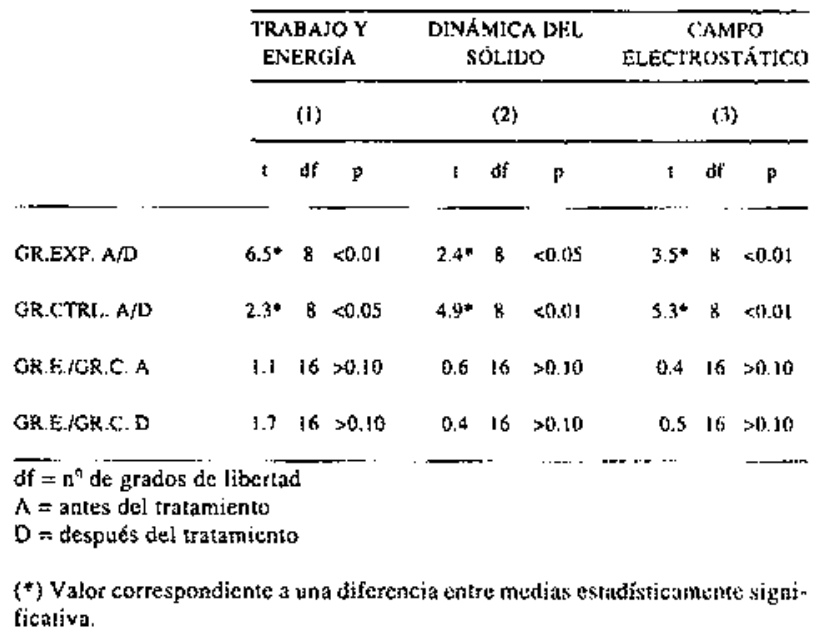

de aprendizaje se ha producido un cambio sustancial en la estructura conceptual (asociativa) de los estudiantes, cambio que ha sido detectado, siquiera sea groseratmente, a través de los tests de asociación de palabras.

Figura 1

Representación gráfica de la varación de los números de «palabras respuesta» (relacionadas con la "palabra estimulo» correspondiente mediante una ecuación o una cadena de ecuaciones) entre el antes y ei despues del tratamiento para los grupos experimental y de control. Los tres pares de líneas se refieren a los resultados de los respectivos tests

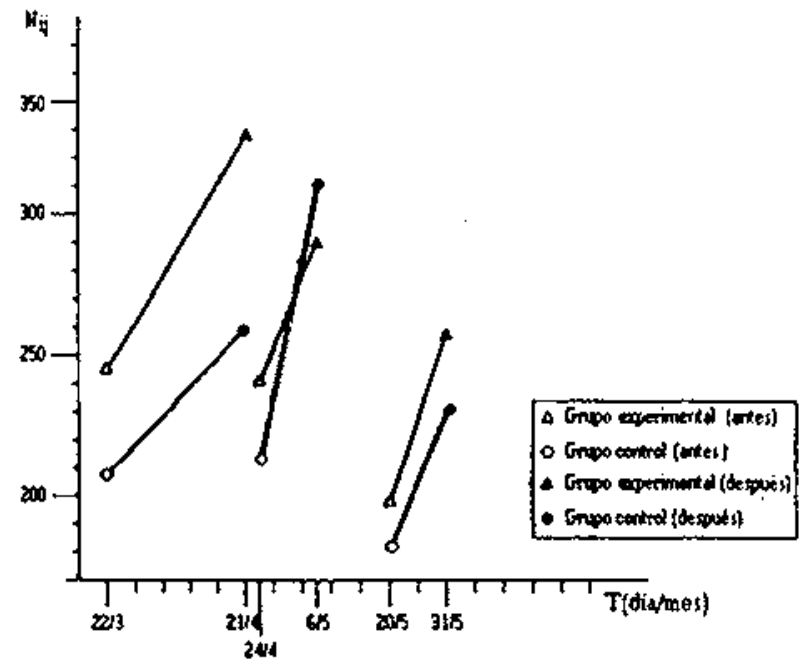

$$
\text { de asociación de palabras. }
$$

\section{Influencia del trabajo personal}

La influencia del trabajo esencialmente personal, orientado a la preparación del examen correspondiente a cada uno de los temas de Física considerados en el presente estudio empírico, se deja sentir con toda claridad en la estructura conceptual (asociativa) de los alumnos, de modo que, como queda reflejado en la figura 1 , tanto el grupo experimental como el de control progresan, en cuanto al número de «palabras respuesta» seleccionadas, en cualquiera de los tres capítulos de Física sobre los que se efectuó el experimento. Además, cuando se considera la significación estadística de las diferencias (Tabla 2) se aprecia que las mismas son, en todos y cada uno de los casos, estadísticamente significativas. Ello implica que la hipótesis nula -que, como es sabido, es el tipo de hipótesis que prueban directamente las técnicas estadísticases rechazada y, por contra, resulta validada la hipótesis alternativa, que ha sido designada con anterioridad como hipótesis 1. Como consecuencia del proceso completo 


\section{Influencia del tratamiento}

I a influencia del tratamiento se advierte "grosso modo» por el carácter divergente de las rectas que, en la figura 1 , unen los puntos correspondientes a los momentos antes/después para los grupos experimental y de control, respectivamente. Tal circunstancia se aprecia en los resultados relativos a los capítulos 1 y 3 , aunque no en los del capítulo 2. No obstante, cuando tal apreciación se somete al rigor del análisis estadístico se advierte que no existen diferencias significativas entre los grupos experimental y de control ni antes ni después del tratamiento en ninguno de los casos considerados. La ausencia de diferencias significativas para los valores iniciales asegura la deseada equivalencia estadística de los grupos experimental y de control. Sin embargo, en el caso de los valores finales, tal circunstancia nos impide rechazar la hipótesis nula planteađa en términos de no diferencias, o lo que es lo mismo, la hipótesis 2 no queda validada por el experimento. Por tanto, a la luz de nuestros resultados no podemos sostener, fundadamente, que la elaboración de mapas conceptuales haya modificado suficientemente el aspecto de la estructura conceptual (asociativa) de los estudiantes que mide los tests de asociación de palabras. Parece como si el tratamiento no afiadiera a dicha estructura mucho más de lo que añade la preparación y el estudio personal, supuestamente intensos, con vistas a la realización de un examen.

\section{DISCUSIÓN}

Del presente experimento se derivan dos conclusiones relativamente claras: la influencia, estadísticamente significativa, del estudio y la preparación personal en la estructura conceptual (asociativa) del alumno, y la influencia no significativa, en tanto que elemento adicional, de la construcción por el alumno del correspondiente mapa conceptual. Además, como tendremos oportunidad de plantear en lo que sigue, la validez de tales conclusiones no resulta independiente una respecto de la otra, sino que están relacionadas entre sí.

A la hora de discutir el efecto de la preparación y estudio personales de cara a la realización de un examen, cabe resaltar tanto su significación estadística como su consistencia a lo largo de las tres ocasiones en las que el experiment o fue repetido. Ambas circunstancias revelan algo ciertamente plausible, a saber, que este tipo de tareas al suponer una profundización, por parte del alumno, en los diferentes elementos temáticos mejoran, cuando menos, la estructura conceptual del sujeto en sus aspectos asociativos. Pero, además, una tal congruencia entre lo previsible y lo resultante constituye un elemento de validación del instrumento y del método de medida. Los tests de asociación de palabras se revelan, por tanto y de nuevo, como una herramienta válida para detectar cierto tipo de cambios en la estructura cognitiva de los alumnos si dichos cambios son de suficiente magnitud.

No es ésta la primera vez que se piantea el problema de la validez de los tests de asociación de palabras. En anteriores investigaciones Shavelson et al. (1975) han establecido la validez de dicha herramienta y otros han contribuido a reforzarla al detectar variaciones significativas en la estructura conceptual (asociativa) de los alumnos como consecuencia de la instrucción (Thro 1978. Shavelson 1972, 1973, Johnstone et al. 1985, Gussarsky ot al. 1988). En la presente investigacion hemos centrado, sin embargo, nuestra atención en un intervalo restringido del proceso de instrucción especialmente significativo en orden a producir alteraciones en el sistema de relaciones conceptuales, dado que conlleva un protagonismo ineludible de la actividad intelectual del sujeto. Los resultados obtenidos contribuyen, pues, a reforzar la validez del instrumento empleado.

Frente a este primer resultado positivo, con respecto a las expectativas resumidas en la hipótesis 1 , el resultado negativo obtenido con relación a la hipótesis 2 requicre un análisis más detallado. Admitiendo, como punto de partida, una postura de extrema confianza en relación con la efectividad de los mapas conceptuales como instrumento de enseñanza/aprendizaje, cabría explicar los resultados negativos del presente estudio situándose en posiciones como las siguientes:
a) Desconfiar del instrumento de medida.
b) Desconfiar de la oportunidad del tratamiento.

En relación con la primera posición es necesario asumir, sin duda alguna, sus limitaciones. Se trata ciertamente de una herramienta que no da información, por ejemplo, de los aspectos proposicionales de la estructura conceptual. Asf, el hecho de que dos series de «palabras respuestas» sean idénticas no supone, necesariamente, que la estructura proposicional sea la misma en ambos casos, e incluso ambas podrían ocuitar errores conceptuales importantes.

La estructura asociativa -que es considerada por los "associative mappers» como un patrón de relaciones entre conceptos establecido en la memoria a largo plazo y que se deriva, en todo o en parte, de las estructuras cognitivas en la memoria semántica (Thro 1978) - constituye, sin lugar a dudas y en el caso que nos ocupa, el aspecto más superficial o laxo de la estructura cognitiva de un sujeto. Una tal limitación -que se hace, por lo demás, evidente ante el corrector de los tests de asociación de palabras cuando dispone de una información derivada del conocimiento personal de los alumnos y de sus actuaciones en contextos de instrucción variados- explica la ya referida crítica de Strike y l'osner (1976) respecto de los tests de asociación de palabras cuando afirman que «no queda claro de qué cosa se está representando su estructura» (p.125). Sin embargo, si fuéramos capaces de desarrollar un test válido y fiable que nos permitiera medir la estructura cognitiva en sus aspectos proposicionalcs habríamos refinado, ciertamente, nuestra aproximación y nos habríamos visto, además, liberados de las connotaciones conductistas de los términos empleados, pero, análogamente a lo que sucedía en el caso anterior, ta existencia de dos estructuras proposicionales idénticas no nos aseguraría, en este caso, un comportamiento análogo de los correspondientes sujetos ante una tarea de resolución de problemas en la que hubiera de intervenir una contribución sustancial de conocimiento procedimental. 
El contenido del conocimiento científico y su organización en la mente de un sujeto constituye una realidad compleja a la que nos aproximamos mediante modelos cognitivos. Pero tales modelos, por su propia naturaleza, constituyen aproximaciones parciales a dicha realidad. Utilizando como imagen la dimensión de la profundidad podríamos postular una progresión de lo superficial a lo profundo según la secuencia: estructura asociativa $\rightarrow$ estructura proposicional $\rightarrow$ estructura procedimental, Sólo los modelos que tomen en consideración una tal dimensión podrán dar cuenta de la complejidad del conocimiento que se pone en jucgo en el aprendizaje científico.

Asumiendo, pues, como limitación el hecho de que los tests de asociacion de palabras se dirijan a los aspectos más laxos $\rightarrow 0$ si se quiere más superficiales- de la organización cognitiva del sujeto, la desconfianza sobre la validez del instrumento de medida no está justificada, como lo prucban los resultados positivos obtenidos en relación con la hipótesis 1 . Los tests de asociación de palabras podrían revelar cambios en la estructura asociativa inducidos por el tratamiento si éstos fueran suficientemente importantes, pero por lo que parece no lo son.

En relación con las características del tratamiento cabe señalar que se ha exigido a los mapas una densidad y una jerarquización adecuada, por una parte, a la riqueza conceptual deI tema y, por otra, al procedimiento de construcción del mapa. La figura 2 ilustra, a modo de ejemplo, uno de los mapas del alumno experimental M.G. A pesar de la indudable calidad del mapa y de tratarse del alumno con mejores resultados académicos, cuando se compara su comportamiento con el de otros no sometidos a tratamiento (Tabla I) no se observa una superioridad sistemática. Del análisis de los diferentes mapas se deduce que el tratamiento fue, en términos generales, como se esperaba que fuera, esto es, tradu. ciéndose en mapas conceptuales suficientemente ricos, complejos y jerarquizados. Es posible, no obstante, poner en duda la intensidad de dicho tratamiento; ésa es, desde luego, otra cuestión que afecta más a la herramienta instructiva y a sus características que a la naturaleza del tratamiento en sí. Un mapa conceptual, si se hace bien, puede llevar al alumno una o dos horas de trabajo personal y es posible que dicho período sea sensiblemente inferior en cantidad e intensidad al que requiere la preparación de un examen; pero como tarea a realizar por el estudiante no da mucho más de sí.

Un escrupuloso defensor de la teoría de Ausubel podría, además, argumentar que los mapas empleados no respetan, estrictamente hablando, la idea de inclusividad conceptual que constituye un elemento fundamental de dicha teoría. La realidad es que en absoluto la rechazan. Así, por ejemplo, en el tema de Trabajo y Energia, en el que se adoptó una estrategia didáctica consistente en considerar la noción de cnergía como primitiva (López.

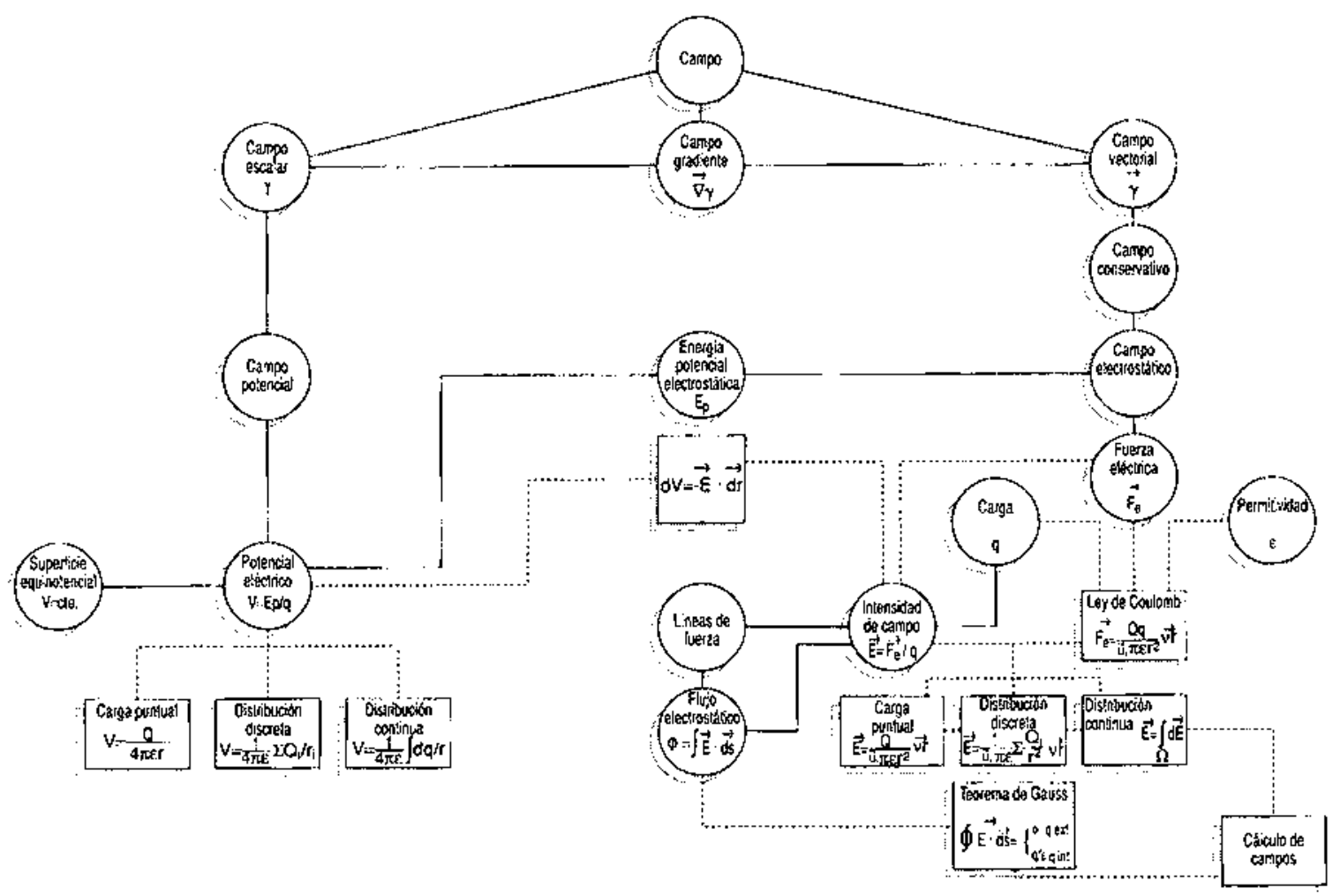


Rupérez et al. 1983, Hierrezuelo et al. 1990), los mapas conceptuales resultantes se aproximaron bastante a los de tipo Novak y es que, orientando el enfoque de este modo, el concepto de energía es más general -en el sentido de más inclusivo- que el de trabajo o que los de energía potencial y cinética, por ejemplo, apareciendo, por lo tanto, situado dentro del mapa en el nivel más elevado de la jerarquía.

Cabe efectuar, finalmente, un análisis crítico del experimento y de sus resultados tomando en cuenta la naturaleza de las variables consideradas. Con todas las limitaciones derivadas del carácter restringido de la muestra, parece que la construcción del mapa conceptual por parte de los alumnos, tras una instrucción completa, no influye significativamente en los aspectos asociativos de la estructura conceptual del individuo. Como hemos señalado antes, una misma estructura asociativa no supone, necesariamente, una misma estructura proposicional o de significado y muy bien pudiera ser que la construcción del mapa conceptual mejorara ćsta sin alterar apreciablemente aquélla.

\section{NOTAS}

(1) Para una discusión más detallada sobre sus limitaciones ver López Rupérez. 1989.

\section{REFERENCIAS BIBLIOGRÁFICAS}

BASCONES, J, y NOVAK, J.D., 1985. Altemative instructional system and the development of problem-solving skills in physics, European Journal of Science Education, $7(3)$, pp. 253-261.

CAMPBELL, D. y STANLEY, I., 1973. Diseños experimentales y cuasiexperimentales en la investigación scial. (Amorrortu: Buenos Aires).

EYLON, B. y REIF, F., 1984. Effects of knowledge Organization in Task Performance. Cognition and Instruction, $1(1)$, pp. 5-44.

GAGNE, R. M., 1971. Las condiciones del aprendizaje. (Aguilar: Madrid).

GORODETSKTY, M. y GUSSARSKY, E., 1986. Misconceptualization of the chemical equilibrium concept as reflected via different evaluation methods, European Journal of Science Education, 8(4), pp. 427-441.

GUNSTONE, R. F., 1980. Word association and the destription of cognitive structure, Research in Science Education, 10, pp. $45-54$

GUSSARKY, F. y GORODETSKY, M., 1988. On the chemical equilibrium concept: Constrained word associations and conception, Journal of Research in Science Teaching, 25(5), pp. $319-333$
Como instrumento derivado de la teoría de Ausubel, el mapa conceptual tipo Novak pretende propiciar un aprendizaje significativo mejorando el conocimiento sobre las relaciones entre los conceptos. Un aprendizaje memorístico suficientemente intenso podría traducirse en una secuencia numerosa de «palabras respuesta» ante una «palabra estímulo» dada, sin suponer por ello un conocimiento preciso de su significado dentro de un sistema conceptual de naturaleza relacional como lo es el que corresponde particularmente a la Física. Un planteamiento similar puede ser aplicado al tipo de mapas conceptuales utilizados en la presente investigación, según la cual la construcción del mapa parece añadir muy poco al proceso completo de instrucción (incluido el estudio personal) en lo que respecta a su influencia sobre los aspectos asociativos de la estructura conceptual. Sin embargo, su posible influencia sobre los aspectos proposicionales o de significado constituye una cuestión abierta cuya respuesta pasa, necesariamente, por la elaboración de instrumentos capaces de captar una tal dimensión del conocimiento científico en la memoria del estudiante.

(2) Agradezco al asesor su recomendación de hacer explícito este comentario.

HIERREZUELLO, J. y MOLINA (GONLÁLE7, R., 1990. Una propuesta para la introducción del concepto de energía en Bachillerato, Enseñanza de las Ciencias, 8(1), pp. 23-30.

JOHNSTONE, A. If y MOYNIHAN, T.F., 1985. The relation ship between performance in word association tests and achievement in chemistry, European Journal of Science Education, 7(1), pp. 37-66.

KEMPA, R. F. y NICHOLLS, C.E., 1983. Problem-Solving ability and cognitive structure: an exploratory investigation, European Journal of Science Fducation , 5(2), pp. 171-184.

KEMPA, R.F., 1986. Resolución de problemas de química y estructura cognoscitiva, Enseñanza de las Ciencias, 4(2), pp. $99-110$.

LOPEZ RUPERE7, F. y LÓPEZ RUPÉREZ, \&., 1983. Las nociones de trabajo y energía. Análisis conceptual y didáctico. Bordón, 249, pp. 497-506

LÓPEZRUPÉREZ, F., 1987. Cómo estudiar Física (Ministerio de Educación y Ciencia/Vicens-Vives: Barcelona).

LÓPEZ RUPÉREZ, F., 1989. Organización del conocimiento y resolución de problemas en Física. Programa de Ayudas a la Investigación Educativa 1987. Memoria Final de la Investigación. CIDE-MEC. 
LOPEY RUPEREZ, F., 1990. Organización del conocimiento, mapas conceptuales y resolucion de problemas. Ponencia presentada en el VI Enctentro sobre aspectos didácticos en las Enseñanzas Medias. Universidad de Zaragoza (en prensa).

MOREIRA, M. A. y SANTOS, C. A., 1981. The influence of content organization on student's cognitive structure in thermodynamics. Joumal of Research in Science Teaching, $18(6)$, pp. 525-531.

MORFIRA, M. A. y TAKFCO, G. S., 1985. Mapas conceptuales como recursos instruccionales de la enseñanza de la Física, Enseñanza de las Ciencias, número extra, p. 14

MOYNIHAN, T. F', 1981. Word association techniques for the study of cognitive structure of chemical concepts in Scottish Secondary Schools. M. Sc. Thesis, The University of Glasgow.

MOYNIHAN, T.F., 1982. Word association techniques for the study of cognitive structure of chemical concepts in Scottish Secondary Schools. Chemical Edacation Research in Implication for Teaching. Report of a Symposium (Royal Society of Chemistry, London, pp. 79-89).

NOVAK, J. D., 1976. Lnderstanding the learning processes and effectiveness of teaching methods in the clasroom laboratory and field, Science Education, 60(4), pp. 493-512.

NOVAK, J. D, GOWIN, D. B. y JOHANSEN, G. T., 1983. The use of Concept Mapping and knowledge vee mapping with junior High School Science Students, Science Education, 67(5), pp. 625-645.

NOVAK, J. D, y GOWING, D. B., I984. Learning how to learn. Cap. 2. (Cambridge University Press: Cambridge).

NOVAK, J. D., 1985. Metalearning and metaknowledge strategies to help students learn how to learn, in Cognitive Sructure and Conceptual change. L.H.T. West y A.L. Pines (edis.). (Academic Press: Orlando).

NOVAK, J. D., 1988. Constructivismo humano. Un consenso emergente, Enseñanza de las Ciencias, 6(3), pp. 213-223.
PREECE, P.F.W., 1976. Mapping Cognitive Structure: A comparison of methods, Joumal of Edacational Psychology, 68(1), pp. $1-8$.

PRELCE, P. IF. W., 1978. Exploration of Semantic Space: Review of Research on the organization of scientific concepts in Semantic Mcmory Science Education, 62(4), pp. 547-562.

SHAVELSON, R, J., 1972. Some aspects of the correspondence between Content Structure and Cognitive Structure in Physics Instruction, Journal of Eductational Psychology, 63(3), pp. 225-234.

SHAVELSON, R, J., 1973. Learning from Physics Instruction, Journal of Research in Science Teaching, 10(2), pp. 101. 111.

SHA VEI SON, R. J., 1974. Methods for Fxamining Representations of a Subjet-Metter Structure in a Student's Memory, Journal of Research in Science Teaching, 11(3), pp. 231249.

SHAVELSOX, R. J. y STANTOPN, G. C., 1975. Construct Validation: Methodology and Application to three Measures of Cognitive Structure, Journal of Educational Measurement, 12(2), pp. 67-85.

STEWART, $J .$, 1979. Content and cognitive structure: Critique of Assessment and Representation Techniques used by Science Education Researches, Science Education, 63(3), pp. 395405.

STRIKF, K. A y POSNER, G. J., 1976. Epistemological perspectives on conceptions of curriculum organization and learming, in Review of Research in Education. S.Shulman(ed.) F. F. PEACOCK, Itaka, Il.

STUART, H.A,, 1985. Should concept maps be scored numerically?, European Journal of Science Edtcation, 7(1), pp. 73-81.

TRHO, M. P., 1978. Relationships between Associative and Content Structure of Physics Concepts, Journal of Educational Psychology, 70(6), pp. 971-978. 
ANEXO 1
Relación de las diferentes series de «palabras estímulo» presentadas a los alumnos en las correspondientes pruebas.

TRABAJO Y ENERGÍA

Posición
Velocidad
Fuerza
Potencia
Energía cinética
Energía potcncial
Energía
Trabajo

\section{DINÁMICA DEL SÓLIDO}

Ángulo de rotación

Momerito de inercia

Velocidad angular

Momerto de la fuerza

Energíc de rotación

Trabajo de rotación

Momento angular

Aceleración angular

\section{CAMPO FLECTROSTÁTICO}

Carga cléctrica

Intensidad de campo

Potencial

Gradiente

Trabajo eléctrico

Fuerza electrostática

Permitividad dicléctrica

Encrgía potencial electrostática

\section{ANEXO 2}

Hoja de instrucciones correspondiente al primer test de asociación de palabras aplicado que fue omitido posteriormente en tests sucesivos. (Aclaptado de Shavelson 1974).

\section{HOJA DE INSTRUCCIONES}

Esta prueba es un test de asociación de palabras que pretende determinar cuántas palabras puedes pensar y escribir, a partir de una dada, en un intervalo de tiempo corto. En la parte superior de una hoja se te presentará una palabra clave que representa un concepto de Fisica y deberás escribir debajo todas aquellas palabras que te vengan a la mente a partir de la palabra clave. Disponúrás de otras tantas hojas para otras tantas palabras clave. Probablemente no seas capaz de rellenar todos los espacios de cada página, pero intenta hacerto lo mejor posible.

A modo de ejemplo, supón que se pide a un electricista que escriba tantas palabras rclacionadas con la electricidad, que es su especialidad, como sea capaz de pensar a partir de la palabra «conductor». Probablemente escribiría lo siguiente:

\section{CONDUCTOR}

metal

resistencia

Conductor

Conductor

carga

Conductor

Conductor

transmite

Conductor

Conductor

Como se trata de un electricista ffjate que no ha escrito las palabras «tren», ni «trolebús», ni «guía», por ejemplo, pues no están relacionadas con el concepto electrico de «conductor». En la situación real deberás pensar, en este caso, como un físico cuando vayas a realizar la prueba. Dispondrás de un minuto para cada página, pero no pases a la siguiente hasta que no se te avise. 


\section{ANEXO 3}

Conjunto de normas dictadas a los alumnos para la elaboración de mapas conceptuales jerárquicos. La aplicación de tales normas fúe ilustrada sobre un ejemplo práctico (López Rupérez 1987).

a) Elabora una lista de conceptos relativos al tema en cuestion tan detallada como te sea posible.

b) Haz otra lista con las leyes, los teoremas y las consecuencias que aparecieron o se emplearon en el desarrollo del tema.

c) Intenta integrar todo lo anterior en un mapa:

i) Reflejando una cierta jerarquía lógica entre conceptos más generales y conceptos derivados de los anteriores por definición.

ii) Diferenciando las distintas relaciones entre los conceptos según están vinculados entre sí por definición (utilizando líneas continuas, por ejemplo) o lo están por deducción, por descubrimiento o por experimentación (utilizando líneas de trazos, por ejemplo).

iii) Distinguiendo entre los nudos de la red propiamente dichos (conceptos) y la concreción de las relaciones entre cllos, ya sean de carácter deductivo (teoremas o consecucncias), ya sean de carácter empírico (leyes).

d) Con el propósito de facilitar la funcionalidad del esquema escribe en todos los casos, junto con el nombre del concepto o de la relación, su símbolo y su expresión maternática, si la hubiere. 